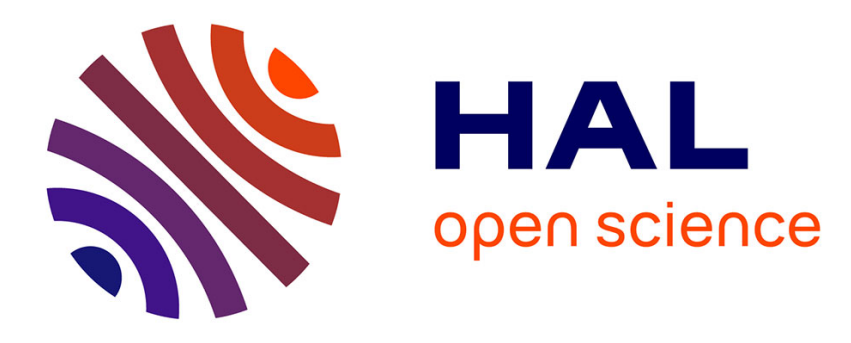

\title{
New Dynamic Muscle Fatigue Model to Limit Musculo-Skeletal Disorder
}

Deep Seth, Damien Chablat, Bennis Fouad, Sophie Sakka, Marc Jubeau, Antoine Nordez

\section{- To cite this version:}

Deep Seth, Damien Chablat, Bennis Fouad, Sophie Sakka, Marc Jubeau, et al.. New Dynamic Muscle Fatigue Model to Limit Musculo-Skeletal Disorder. Laval virtual, Mar 2016, Laval, France. hal01309156

\section{HAL Id: hal-01309156 https://hal.science/hal-01309156}

Submitted on 28 Apr 2016

HAL is a multi-disciplinary open access archive for the deposit and dissemination of scientific research documents, whether they are published or not. The documents may come from teaching and research institutions in France or abroad, or from public or private research centers.
L'archive ouverte pluridisciplinaire HAL, est destinée au dépôt et à la diffusion de documents scientifiques de niveau recherche, publiés ou non, émanant des établissements d'enseignement et de recherche français ou étrangers, des laboratoires publics ou privés. 


\section{New Dynamic Muscle Fatigue Model to Limit Musculo-Skeletal Disorder *}

\author{
Deep Seth \\ IRCCyN, Nantes, France \\ Deep.Seth@irccyn.ec- \\ nantes.fr
Sophie Sakka
IRCCyN, Nantes, France
Sophie.Sakka@irccyn.ec- nantes.fr

\author{
Damien Chablat \\ IRCCyN, Nantes, France \\ Damien.Chablat@irccyn.ec- \\ nantes.fr
}

\author{
Marc Jubeau \\ STAPS, Nantes, France \\ Marc.Jubeau@univ- \\ nantes.fr
}

\author{
Fouad Bennis \\ IRCCyN, Nantes, France \\ Fouad.Bennis@ec- \\ nantes.fr
}

\author{
Antoine Nordez \\ STAPS, Nantes, France \\ Antoine.Nordez@univ- \\ nantes.fr
}

\begin{abstract}
Muscle fatigue is one of the reason leads to Musculo-Skeletal Disorder(MSD). Automation in today's industries makes human effort very less, but still there are many industries in which human have to do complex and repetitive tasks manually. The society/companies have to pay attention on this issue due to the new laws on penibility or repetitive tasks. The objective of this paper is to experimentally validate a new dynamic muscle fatigue model using electromyography (EMG) and Maximum voluntary contraction (MVC). A new model is developed by introducing a co-contraction factor ' $n$ ' in the Ruina Ma's dynamic muscle fatigue model. The experimental data of ten subjects are used to analyze the muscle activities and muscle fatigue during extension-flexion motion of the arm on a constant absolute value of the external load. The findings for co-contraction factor shows that the fatigue increases when co-contraction area decreases. The dynamic muscle fatigue model is validated using the MVC data, fatigue rate and co-contraction factor of the subjects.
\end{abstract}

\section{Keywords}

Muscle fatigue, maximum voluntary contraction (MVC), muscle fatigue model, co-contraction, fatigue rate, electromyography (EMG)

\section{INTRODUCTION}

In the field of industrial bio-mechanics, the fatigue is defined as "any reduction in the maximal capacity to generate the force and power output". In industries, mostly repetitive manual tasks tends to work related MSD problems [15, 17]. Some times people have to work more on the same repetitive task which can be painful [5, 6] and leads to MSD [1, 16] due to muscle fatigue [15]. To avoid

\footnotetext{
* (This work is supported by Erasmus Mundus HERITAGE Project funded by the European Commission and coordinated by Ecole Centrale de Nantes, France).
}

VRIC 16, March 23-25, 2016, Laval, France

- X-XXXXX-XX-X/XX/XX.

DOI: $10.1145 / 1235$
MSD, the study of muscle fatigue is very important. Various static and dynamic muscle fatigue models are proposed earlier to study muscle fatigue $[2,4,9,18,19,20]$. Liang's fatigue model [9] have experiment validation for fatigue and effect of recovery in arm with static drilling posture. Silva [13] simulate the hill model and validate it using opensim. Some Dynamic fatigue models are also introduced [3, 7, 11]. A Dynamic Muscle Fatigue Model [12] has been proposed to describe the fatigue process of muscle groups. However, no consideration about the co-contraction of paired muscles is taken. Missenard [14] explains the effect of fatigue and co-contraction on the accuracy of arms motion. However, no consideration about the co-contraction of paired muscles is taken. The main objective of this study is to revise this dynamic muscle fatigue model by including the factor of co-contraction of paired muscles, as well as to validate it through mathematics and experiments. For now in this article, we mainly focus on the study of muscle cocontraction activity, using elbow joint's muscle groups as target. With the assistance of EMG, the function of co-contraction is confirmed and calculated.

\section{PROPOSED DYNAMIC MODEL OF MUS- CULAR FATIGUE}

The dynamic muscle fatigue model is applicable on the dynamic motion of the human body parts.A dynamic muscle fatigue model is proposed by Liang Ma [9] [8] firstly applied on static drilling task. Ruina Ma [11][12] develops this model for the dynamic motions like push/pull operation of the arm. However, the co-contraction of the muscles are not included in both the models. In dynamic muscle fatigue model [10], we select two parameters $\Gamma_{j o i n t}$ and $\Gamma_{M V C}$ to build our muscle fatigue model. The hypotheses can then be incorporated into a mathematical model of muscle fatigue which is expressed as follows:

$$
\frac{d \Gamma_{c e m}(t)}{d t}=-k \cdot n \cdot \frac{\Gamma_{c e m}}{\Gamma_{M V C}} \Gamma_{j o i n t}(t)
$$

where, $k$ is the fatigue factor and $n$ is the co-contraction factor. And, if $\Gamma_{\text {Joint }}$ and $\Gamma_{M V C}$ held constant, the model can then simplify as follows:

$$
\Gamma_{c e m}(t)=\Gamma_{M V C} \cdot \mathrm{e}^{-k \cdot n \cdot C t}, \quad\left\{\text { where }, \quad C=\frac{\Gamma_{\text {Joint }}}{\Gamma_{M V C}}\right\}
$$




$$
k=\frac{-1}{n \cdot C t} \cdot \ln \left(\frac{\Gamma_{c e m}(t)}{\Gamma_{M V C}}\right)
$$

The other parameters for this model are same as in table 1. $n$ is co-contraction factor.

\begin{tabular}{|l|l|l|}
\hline Elements & Unit & Description \\
\hline \hline$k$ & $\min ^{-1}$ & Fatigue factor, constant \\
$\Gamma_{M V C}$ & N.m & Maximum torque on joint \\
$\Gamma_{\text {Joint }}$ & N.m & Torque from external load \\
$\Gamma_{\text {cem }}$ & N.m & Current capacity of the muscle \\
\hline
\end{tabular}

Table 1: Parameters of dynamic muscle fatigue model

\subsection{Co-contraction factor ' $n$ '}

The co-contraction is the simultaneous contraction of both the agonist and antagonist muscle around a joint to hold a stable position at a time. Assumptions made for finding co-contraction factor are as follows:

1. The co-contraction is the common intersecting area between the two group of acting muscles.

2. The co-contraction factor will be the same for each agonist and antagonist activities.

The co-contraction area can be understand by the figure 1 . This figure is just an example representation of a motion cycle. In this figure, we can see the common EMG activity between bicep and tricep muscle shown by the orange color, which is co-contraction area $C_{A}$. The formula for calculating the co-contraction area from EMG activities is given in equation 4 . The trapezius activity shown along with the two muscles is co-activation.

$$
\begin{gathered}
C_{A}=\frac{\int_{t_{0}}^{t_{100}} E M G_{\text {min }} \times d t}{\int_{t_{0}}^{t_{100}}\left[E M G_{\text {agonist }}+E M G_{\text {antagonist }}\right] \times d t} \\
n=1-C_{A}
\end{gathered}
$$

The co-contraction area $C_{A}$ can also be represented as follows: $C_{A}=$ common activities between the two group of muscles.

$$
C_{A}=a \cdot \exp b \cdot x
$$

where, $a$ and $b$ are constant parameters and $x$ is the time.

where, $E M G_{\text {min }}$ is the common area share by the EMG activity of bicep and tricep, $E M G_{\text {agonist }}$ and $E M G_{\text {antagonist }}$ are the full activities of the bicep and triceps muscle's. The activities of the both the muscles are normalized with respect to the maximum value of the activities of the same muscle.

\subsection{Push-Pull Operation and Muscles activi- ties}

The push/pull motion of the arm is the flexion and extension of the arm about the elbow. The plane of the motion is vertical plane. The Push/pull activities with the muscle activation is shown in figure 2. In Ma's model there were no part of co-contraction and delay in the model which we have added in this new model.

\section{DATA PROCESSING AND METHODOL- OGY}

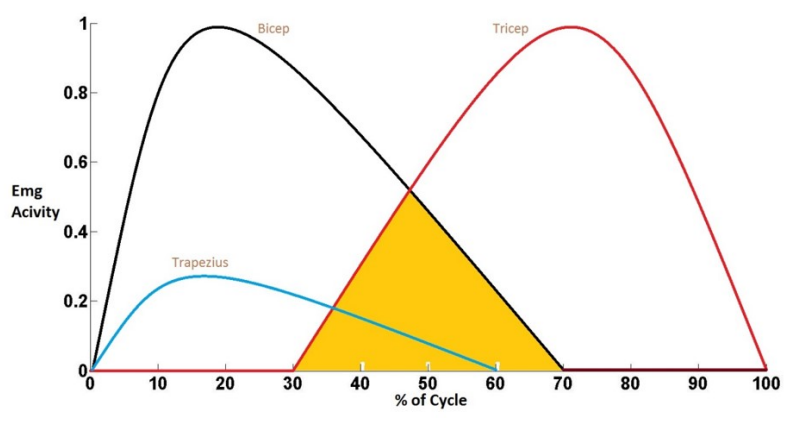

Figure 1: A representative plot of EMG activity of bicep, triceps and trapezius normalized with the maximum value of each muscle's activity for one cycle

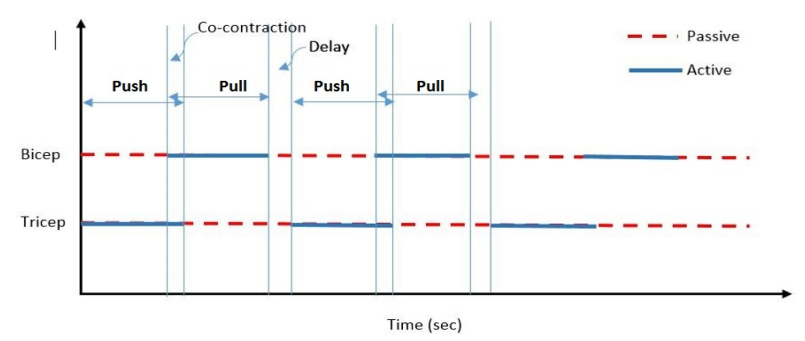

Figure 2: Push/Pull Motion and Muscles activities

\subsection{Experiment Protocol}

- The repetitive flexion and extension of the arm in a vertical plane as shown in figure 3

- The motion range is seventy degrees. The test repetition protocol continues till exhaution.

- Each cycle (flexion + extension) should be completed in 3 seconds.

- External load was $20 \%$ of MVC. MVC test every one minute.

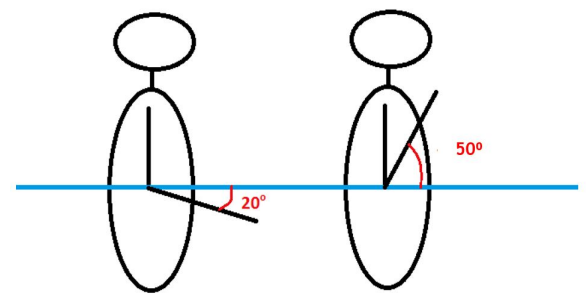

Figure 3: Arm movement range while flexion and extension in vertical plane

\subsection{Data Acquisition}

A Biodex system 3 research (Biodex medical,shirley, NY) isokinetic dynamo-meter was used to measure the value of elbow angle, velocity and torque. The Electromyographic sensor electrodes were put on Biceps, Tricep and Trapezius muscles to record their electrical activities. The frequency of data acquisition was set at $2000 \mathrm{~Hz}$ so that most of the activities get recorded. 


\subsection{Subject's description}

The subjects (all male) details are given in the table 2. All the subjects were sportive.

\begin{tabular}{|l|l|l|c|c|c|c|}
\hline S & Age & Wt. & Height & U. Arm & F.arm & Sport \\
\hline \hline 1 & 28 & $89 \mathrm{~kg}$ & $185 \mathrm{~cm}$ & $29 \mathrm{~cm}$ & $26.5 \mathrm{~cm}$ & Running \\
2 & 24 & $80.2 \mathrm{~kg}$ & $183.5 \mathrm{~cm}$ & $31.5 \mathrm{~cm}$ & $28 \mathrm{~cm}$ & Gym \\
3 & 20 & $69.8 \mathrm{~kg}$ & $180.1 \mathrm{~cm}$ & $30 \mathrm{~cm}$ & $29.5 \mathrm{~cm}$ & Handball \\
4 & 20 & $80.9 \mathrm{~kg}$ & $177 \mathrm{~cm}$ & $29.8 \mathrm{~cm}$ & $29 \mathrm{~cm}$ & Handball \\
5 & 21 & $62.2 \mathrm{~kg}$ & $172.8 \mathrm{~cm}$ & $29.2 \mathrm{~cm}$ & $26.5 \mathrm{~cm}$ & Tennis \\
6 & 25 & $61.1 \mathrm{~kg}$ & $164.8 \mathrm{~cm}$ & $26 \mathrm{~cm}$ & $24.5 \mathrm{~cm}$ & Rugby \\
7 & 26 & $74 \mathrm{~kg}$ & $176 \mathrm{~cm}$ & $28.5 \mathrm{~cm}$ & $27 \mathrm{~cm}$ & Tennis \\
8 & 27 & $66 \mathrm{~kg}$ & $181 \mathrm{~cm}$ & $29.5 \mathrm{~cm}$ & $26.5 \mathrm{~cm}$ & wall climb \\
9 & 23 & $66.3 \mathrm{~kg}$ & $164 \mathrm{~cm}$ & $27 \mathrm{~cm}$ & $25.5 \mathrm{~cm}$ & Swimming \\
10 & 26 & $85 \mathrm{~kg}$ & $184 \mathrm{~cm}$ & $29 \mathrm{~cm}$ & $26.5 \mathrm{~cm}$ & Football \\
\hline
\end{tabular}

Table 2: Subjects anthropometric data and description

\subsection{Data Processing and analysis}

All the raw data were processed using standardized MATLAB program. Data processing includes noise filtering from raw EMG data with the filter frequency $10 \mathrm{~Hz}$ for low pass filter and $400 \mathrm{~Hz}$ for high pass filter and normalization of the data. The total number of cycles compared for all the ten subjects are 1998 cycles. All the cycles are normalized on time scale and compared. The cycle selection for flexion and extension phases is done according to the velocity change in each cycle. The collective EMG plots for Biceps, Triceps and Trapezius muscle are show in figure 4 and figure 5 for all the ten subjects and the collective comparison for the mechanical data position, velocity and torque is shown in figure 6 and figure 7 for all the ten subjects.

For figure4, 5, 6and 7 representations are as follows:

Blue color curve show mean EMG activity.

I Red bar plot on blue curve shows the standard deviation of all the EMG activities along the mean.

- Black dotted curves shows the maximum and minimum reach from the EMG activies. All the cycles are normalized according to the equation:

$$
\text { value }_{\text {Normalization }}=\text { value }_{\text {std }} \text { max }+2 \sigma
$$

value $_{\text {Normalization }}$ : Under it all the muscle activity will be normalized.

value $_{\text {std }}^{\max }$ : Maximum value of standard deviation along the mean. $2 \sigma: \sigma$ values addition upto $2 \sigma$

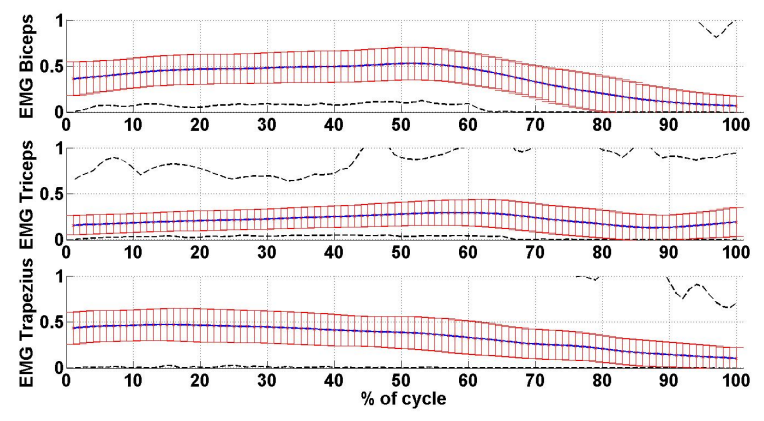

Figure 4: Mean and Standard deviation plots for EMG data of Bicep, triceps and trapezius in flexion phase for all subjects

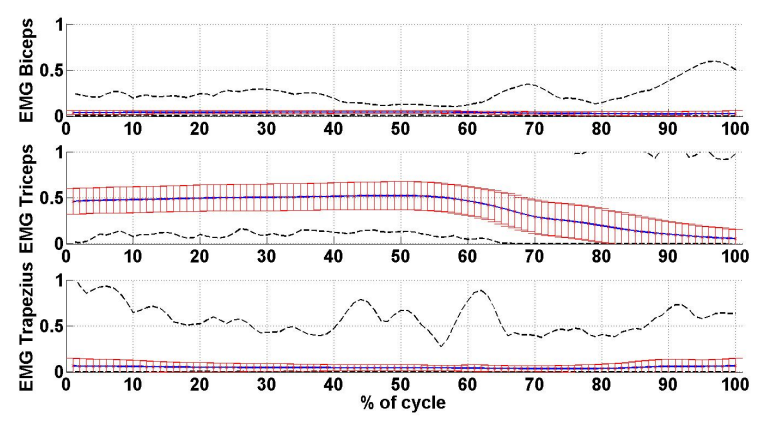

Figure 5: Mean and Standard deviation plots for EMG data of Bicep, Triceps and Trapezius in Extension Phase for all subjects

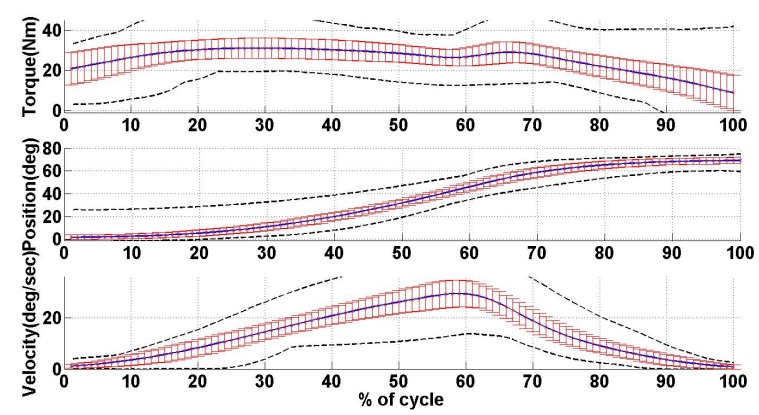

Figure 6: Mean and Standard deviation plots for velocity, position and torque in Flexion Phase for all subjects

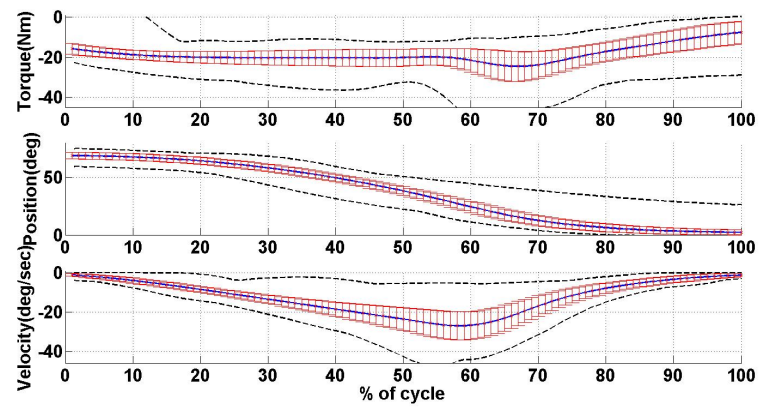

Figure 7: Mean and Standard deviation plots for velocity, position and torque in Extension Phase for all subjects

\section{RESULTS AND DISCUSSION}

The raw data obtained after the fatigue test is processed and the results and observations are discussed in this section. After processing the EMG data of all the muscle groups from figure 4, 5, 6 and 7, we can observe that when the biceps are active during flexion phase there are always some activities from the triceps and on the other hand when triceps are active during pull phase the biceps are almost passive or activities are very near to zero. We can also observe the co-activation of trapezius muscle with the activation of biceps. The activation of triceps with the biceps is co-contraction between two muscles during flexion phase. 
The co-contraction area calculated by using equation 4 is fitted with the exponential equation 6 in section 2.1. The figure $8-17$ shows the fitted graphs for the co-contraction percentage for test cycles of all ten subjects. In figures $8-17$ blue dots show the percentage area of contraction during each extension-flexion cycle and red curve shows the exponential fit for the percentage co-contraction. This shows that the co-contraction percentage for activity between the muscles reduces as the fatigue test proceed or the muscles gets fatigued. By the equation 3 and 4 we can find $n_{i}$ as shown in table 3, where $i$ is the subject number:

\begin{tabular}{|c|c|c|c|c|c|c|c|c|c|c|}
\hline$n_{i}$ & $n_{1}$ & $n_{2}$ & $n_{3}$ & $n_{4}$ & $n_{5}$ & $n_{6}$ & $n_{7}$ & $n_{8}$ & $n_{9}$ & $n_{10}$ \\
\hline \hline$n$ & 0.6 & 0.5 & 0.67 & 0.6 & 0.59 & 0.65 & 0.64 & 0.7 & 0.5 & 0.7 \\
\hline
\end{tabular}

Table 3: Co-contraction factor for each subject

We can notice that only the subject number 8 in figure 15 has increasing slope for the co-contraction area. This behavior can be associated with his sport activity which is wall climbing and very different from other subjects as shown in table 2.

The co-activation of the trapezius muscle is observed mostly in the flexion phase. The MVC values are measured between each protocol of one minute. We can see in most of the cases MVC decreases as fatigue increases. The MVC is same as $\Gamma_{c e m}$ used in our model. The theoretical and experimental evolution of $\Gamma_{c e m}$ is on the basis of $k$ (fatigue rate) using equation 2 and equation 2 and calculated $n_{i}$ and $C=0.2$. The evaluation of fatigue parameter ' $k$ ' for $\Gamma_{\text {cem }}$ extension is shown in figure 18, 20, 22, 24, 26, 28, 30, 32, 34 and 36 . Similarly fatigue parameter ' $k$ ' evaluation for $\Gamma_{c e m}$ flexion is shown in figure $19,21,23,25,27,29,31,33,35$ and 37. In these figures blue line shows the MVC measured for flexion and extension after each test protocol of 1 minute. The theoretical and experimental evolution of $\Gamma_{c e m}$ shows that the experimental values are well fit with in the theoretical model. The co-contraction factor have significant effect on the model. The fatigue rate increases with the input of co-contraction factor. The minimum, maximum and average value of ' $k$ ' for each subject are shown in table 4 .

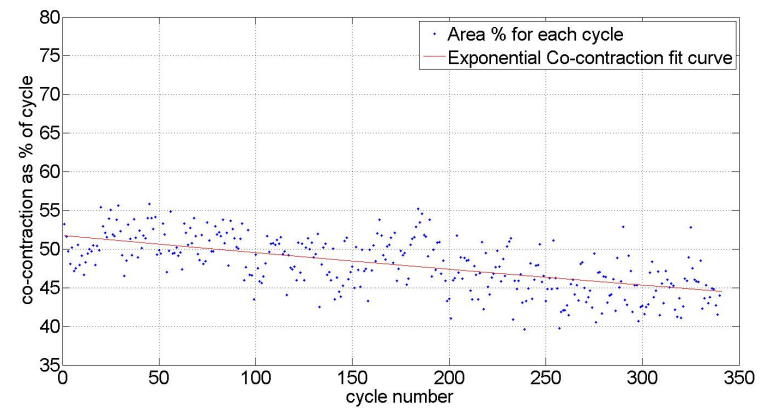

Figure 8: Exponential curve fit of co-contraction area for subject 1

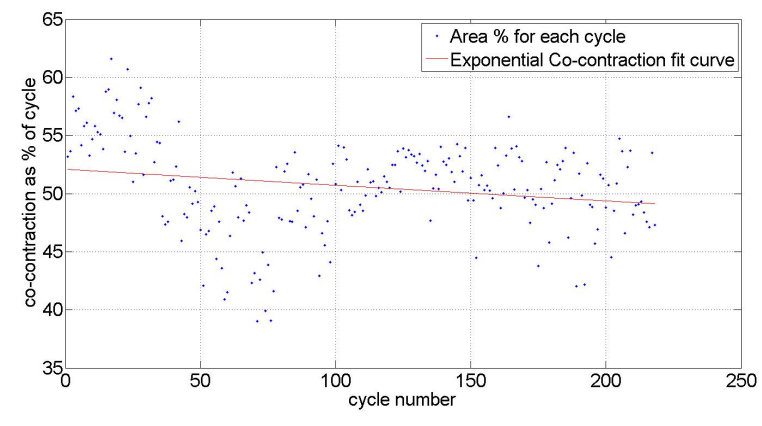

Figure 9: Exponential curve fit of co-contraction area for subject 2

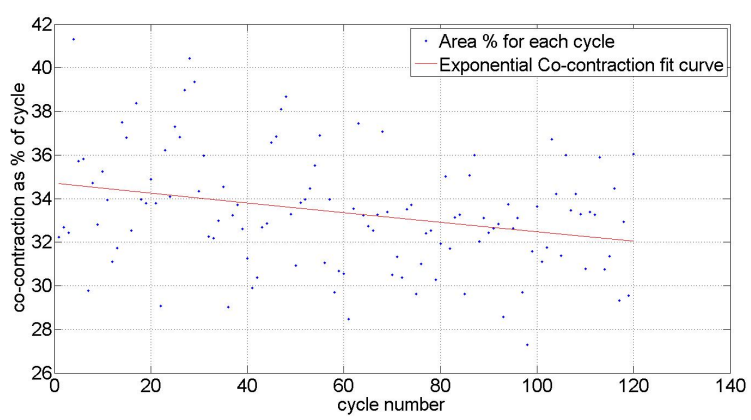

Figure 10: Exponential curve fit of co-contraction area for subject 3

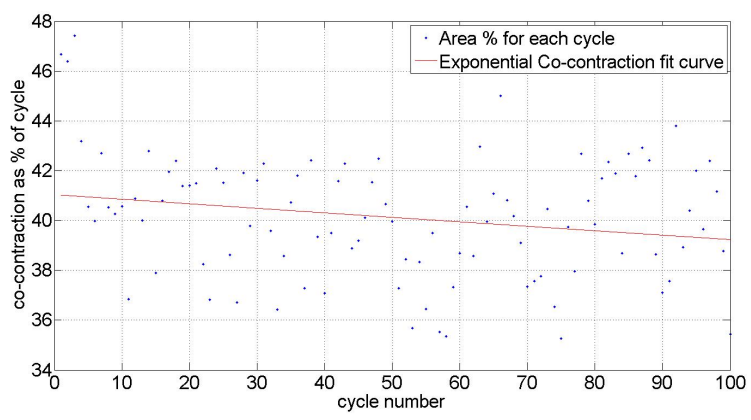

Figure 11: Exponential curve fit of co-contraction area for subject 4

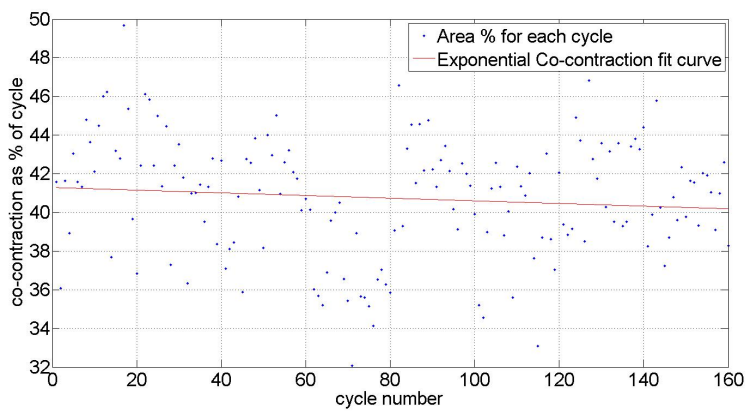

Figure 12: Exponential curve fit of co-contraction area for subject 5 


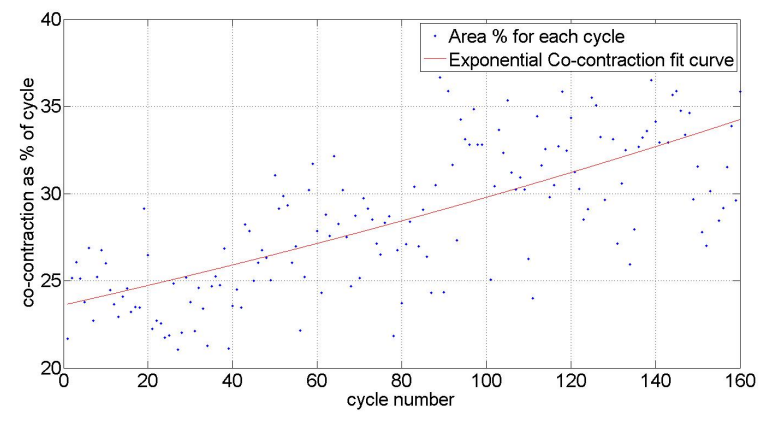

Figure 15: Exponential curve fit of co-contraction area for subject 8

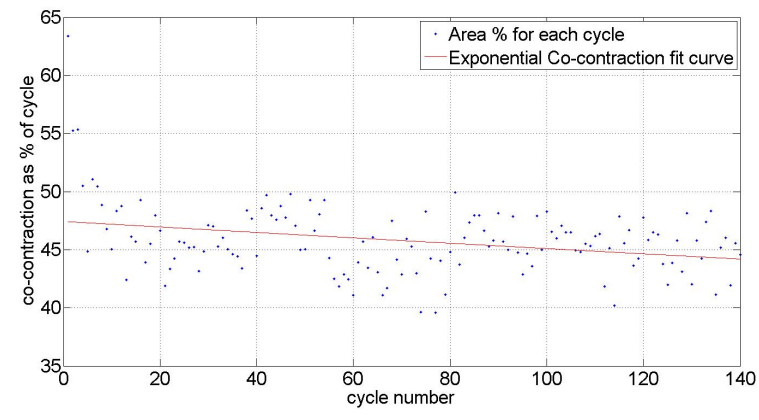

Figure 16: Exponential curve fit of co-contraction area for subject 9

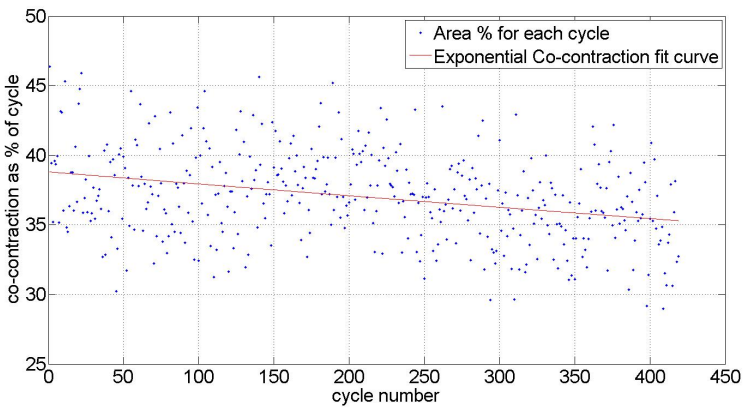

Figure 13: Exponential curve fit of co-contraction area for subject 6

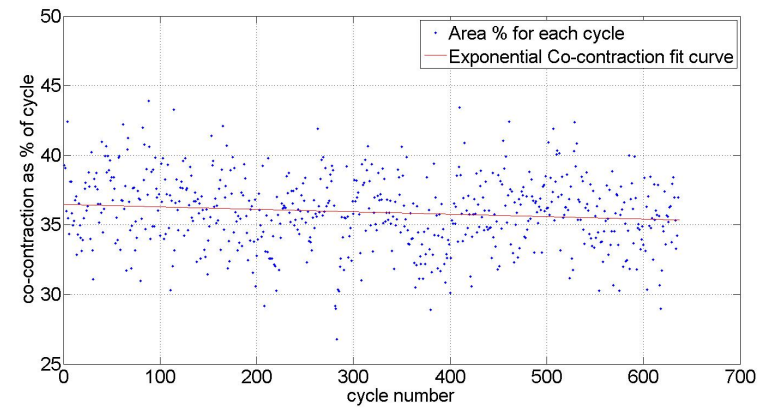

Figure 14: Exponential curve fit of co-contraction area for subject 7

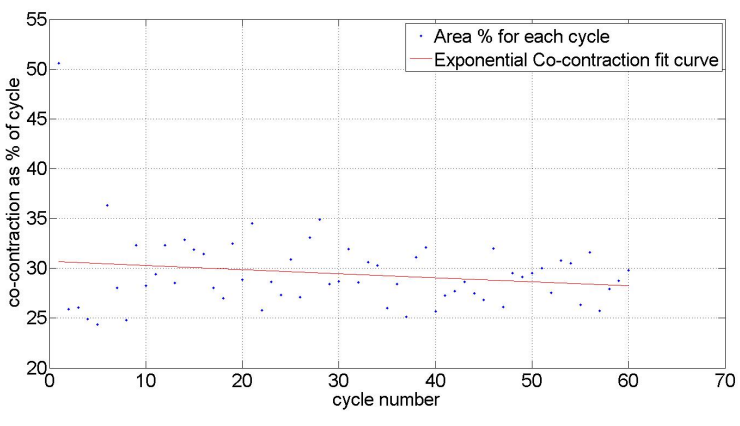

Figure 17: Exponential curve fit of co-contraction area for subject 10

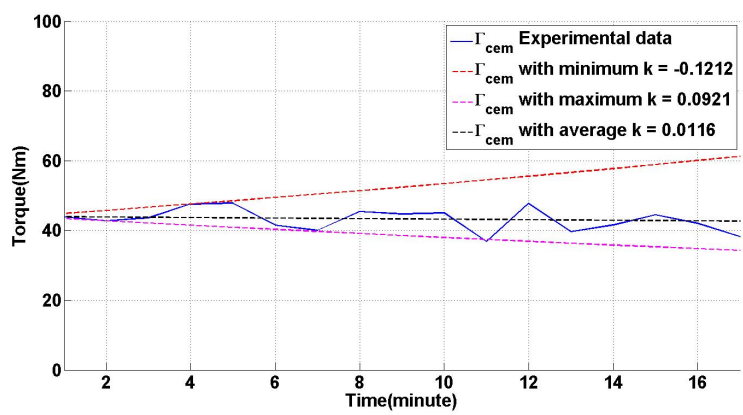

Figure 18: The extension in the subject 1

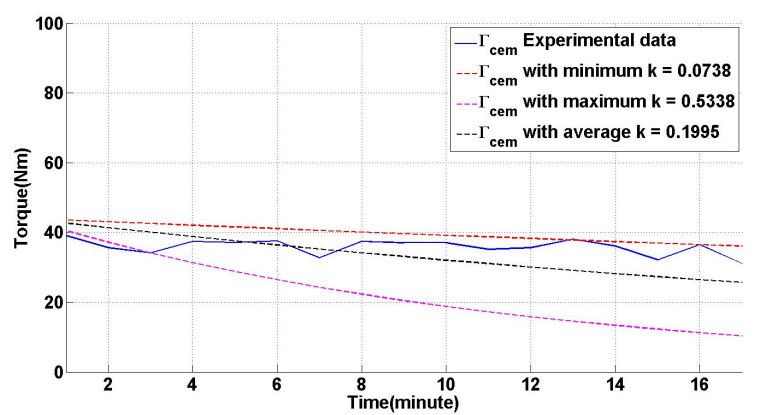

Figure 19: The flexion in the subject 1

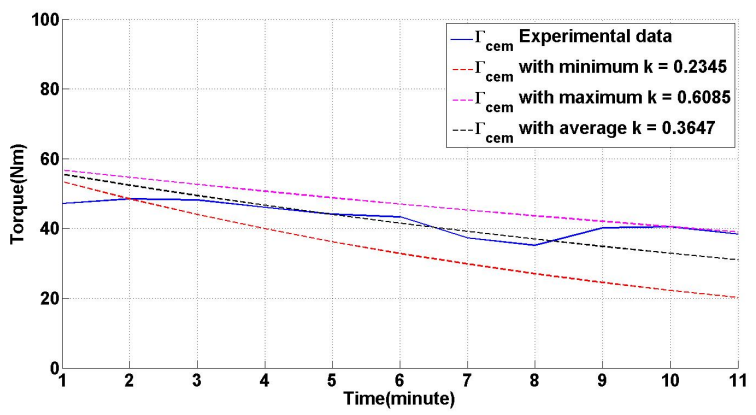

Figure 20: The extension in the subject 2 


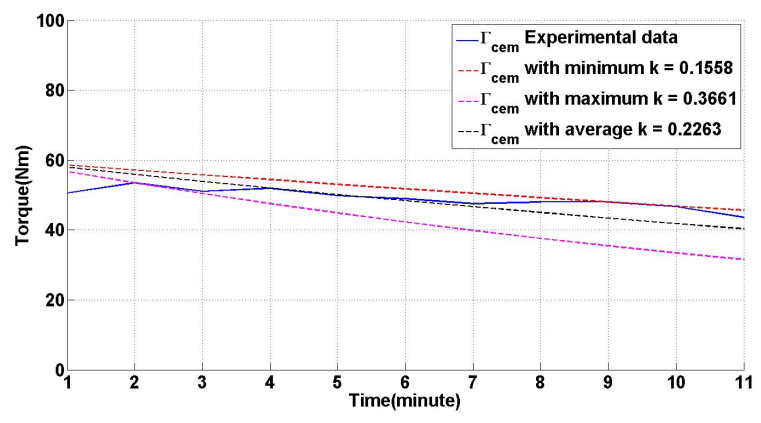

Figure 21: The flexion in the subject 2

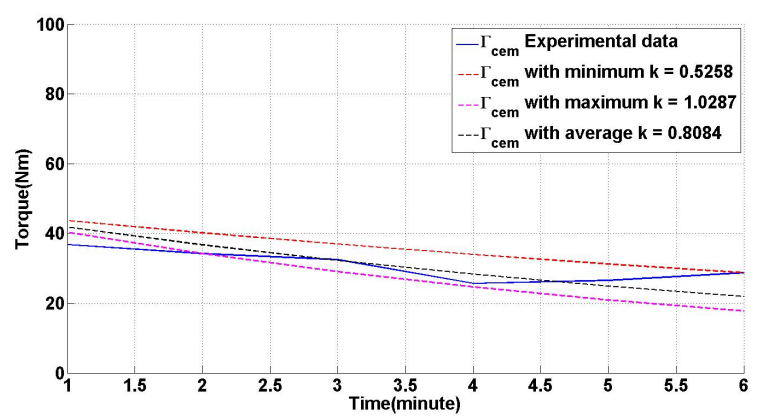

Figure 22: The extension in the subject 3

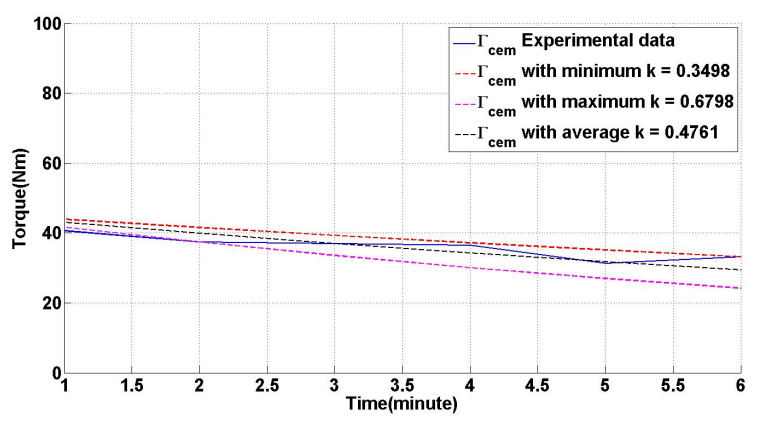

Figure 23: The flexion in the subject 3

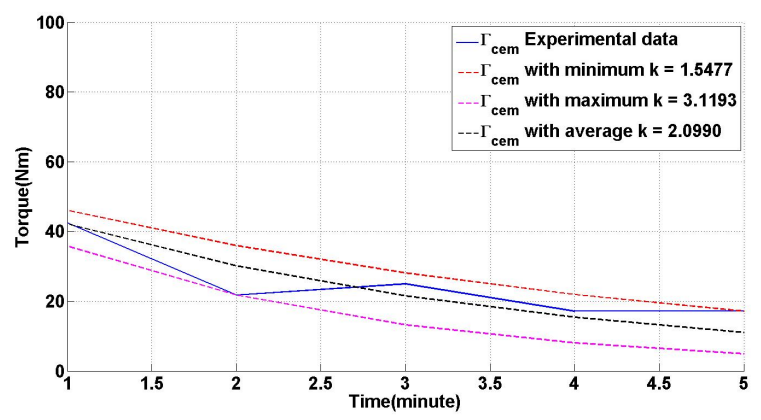

Figure 24: The extension in the subject 4

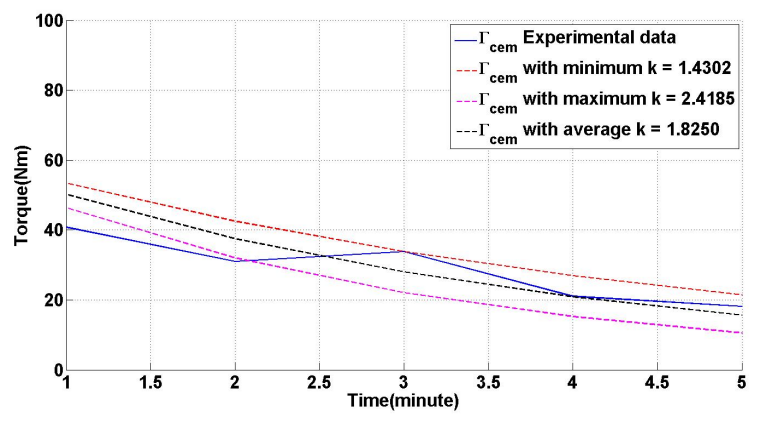

Figure 25: The flexion in the subject 4

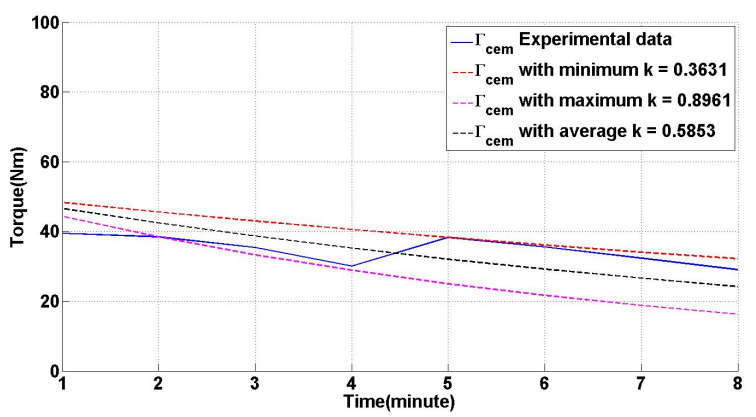

Figure 26: The extension in the subject 5

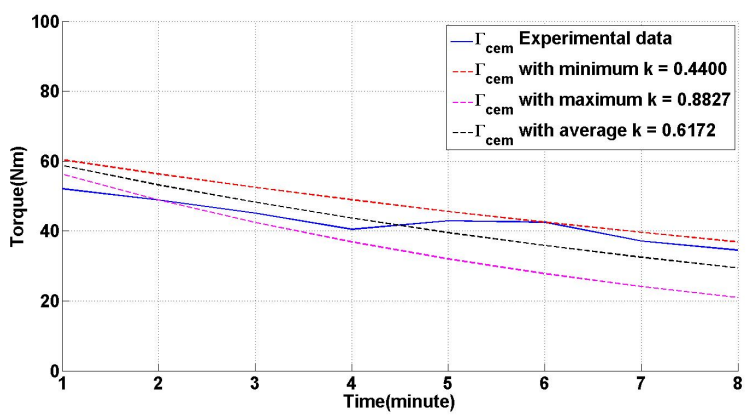

Figure 27: The flexion in the subject 5

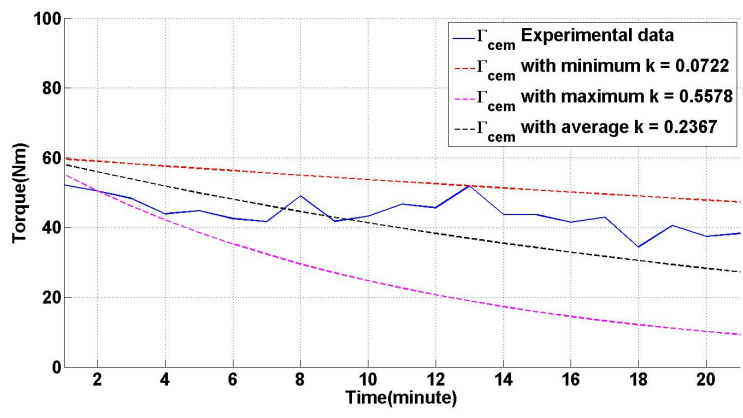

Figure 28: The extension in the subject 6 


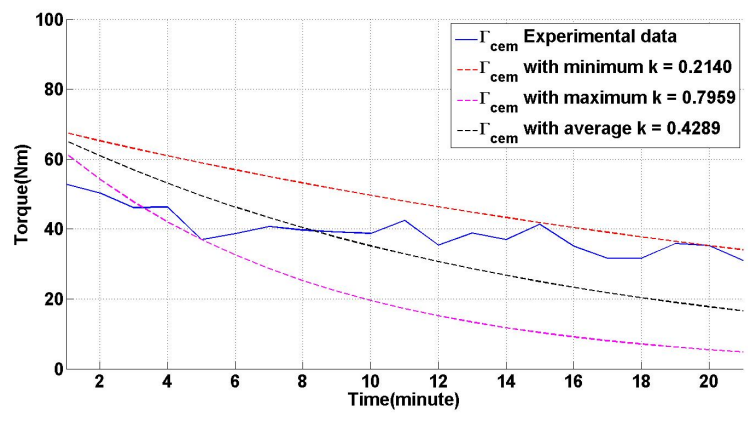

Figure 29: The flexion in the subject 6

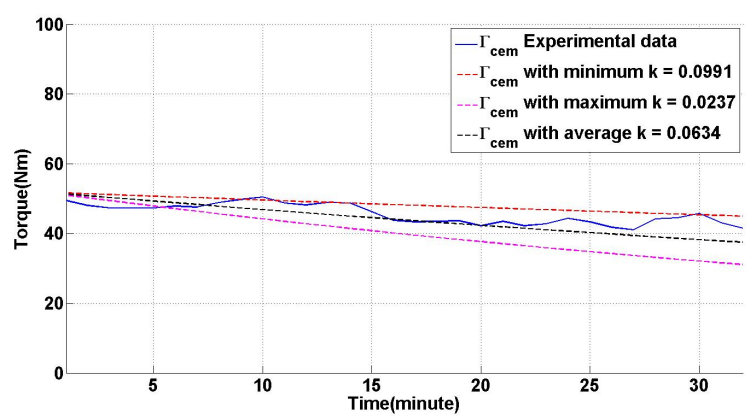

Figure 30: The extension in the subject 7

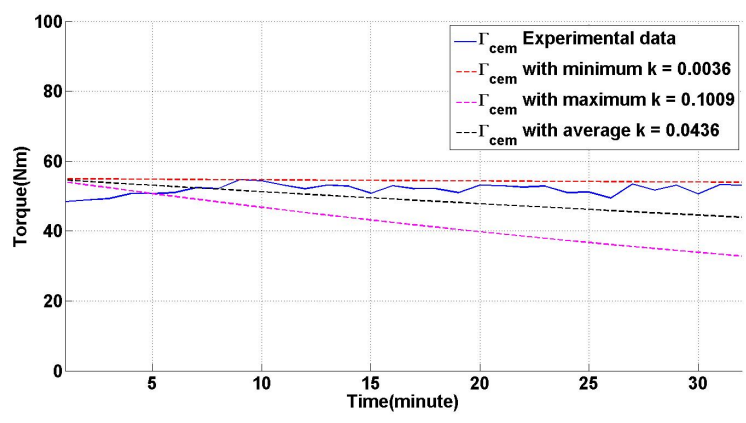

Figure 31: The flexion in the subject 7

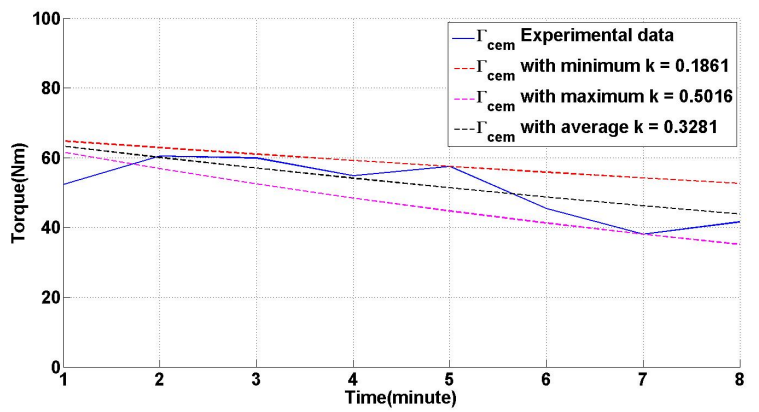

Figure 32: The extension in the subject 8

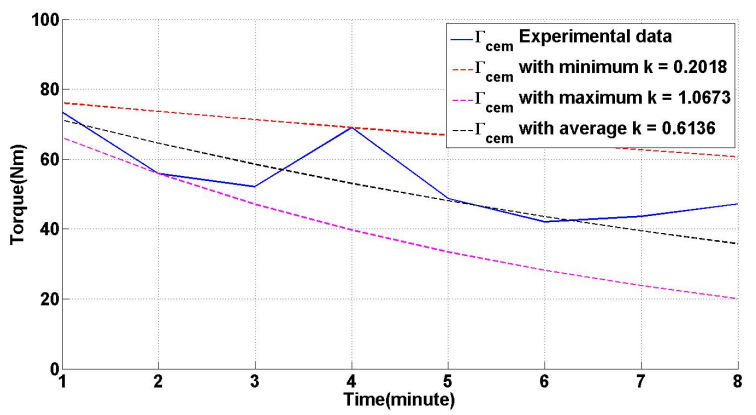

Figure 33: The flexion in the subject 8

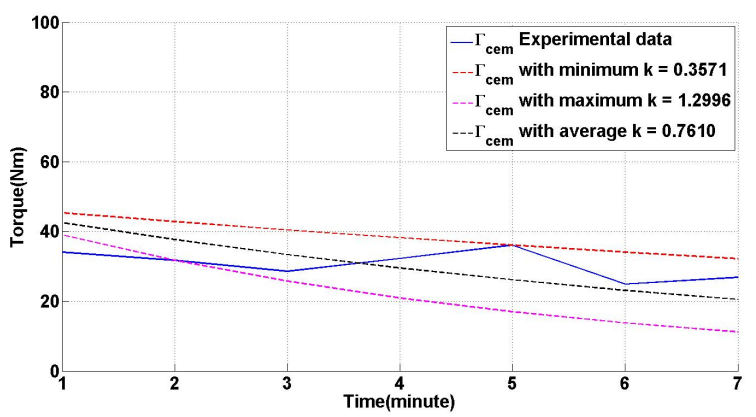

Figure 34: The extension in the subject 9

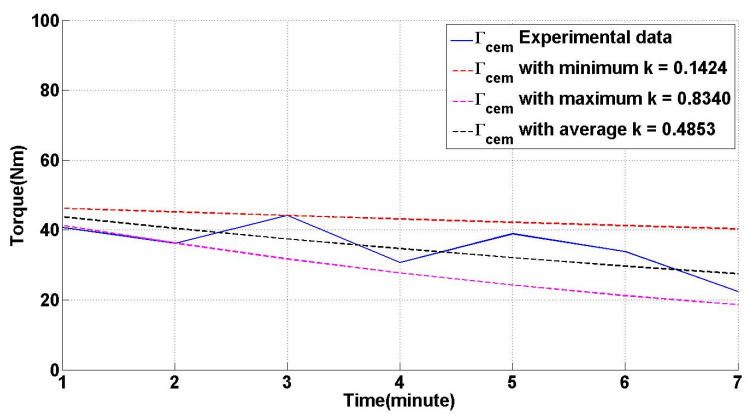

Figure 35: The flexion in the subject 9

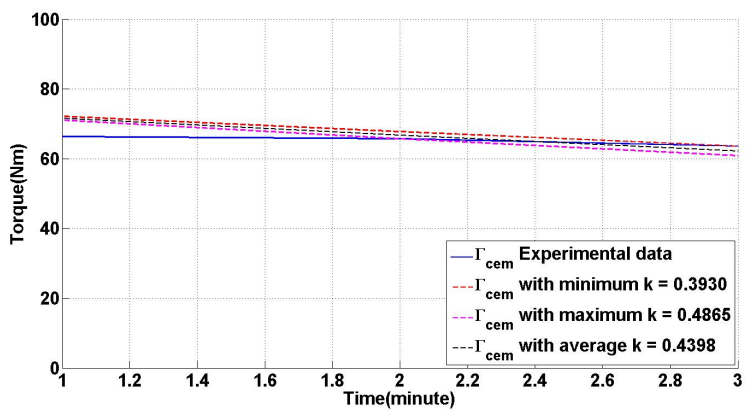

Figure 36: The extension in the subject 10 


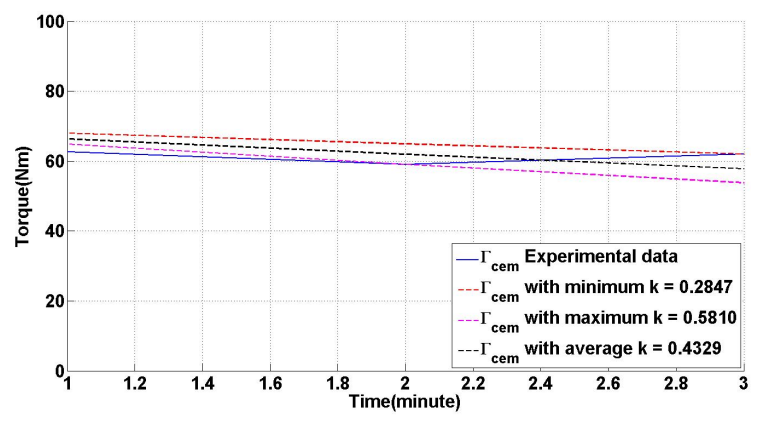

Figure 37: The flexion in the subject 10

\begin{tabular}{|c||c|c|c||c|c|c|}
\hline & \multicolumn{3}{|c|}{$k_{\text {extension }}$} & \multicolumn{3}{c|}{$k_{\text {flexion }}$} \\
\hline S & Min & Max & Avg & Min & Max & Avg \\
\hline 1 & -0.1212 & 0.0921 & 0.0116 & 0.0738 & 0.5338 & 0.1995 \\
2 & 0.2345 & 0.6085 & 0.3647 & 0.1558 & 0.3661 & 0.2263 \\
3 & 0.5258 & 1.0287 & 0.8084 & 0.3498 & 0.6798 & 0.4761 \\
4 & 1.5477 & 3.1993 & 2.0990 & 1.4302 & 2.4185 & 1.8250 \\
5 & 0.3631 & 0.8961 & 0.5853 & 0.4400 & 0.8827 & 0.6172 \\
6 & 0.0722 & 0.5578 & 0.2367 & 0.2140 & 0.7959 & 0.4289 \\
7 & 0.0237 & 0.0991 & 0.0634 & 0.0036 & 0.1009 & 0.0436 \\
8 & 0.1861 & 0.5061 & 0.3281 & 0.2018 & 1.0673 & 0.6136 \\
9 & 0.3571 & 1.2996 & 0.7610 & 0.1424 & 0.8340 & 0.4853 \\
10 & 0.3930 & 0.4865 & 0.4398 & 0.2847 & 0.5810 & 0.4329 \\
\hline
\end{tabular}

Table 4: Experimentally calculated values of ' $k$ ' for flexion and extension motion

\section{CONCLUSIONS}

The proposed model for dynamic muscle fatigue includes the co-contraction parameter, unlike in any other existing model according to the author's knowledge. The results and analysis of the experimental data validates the most of the assumptions made for the proposed model. EMG analysis along with MVC helps to understand the muscle activities, also it justifies the significance of the co-contraction parameter in proposed dynamic muscle fatigue model. The experimental data also helps in validating the new dynamic muscle fatigue model.

\section{ACKNOWLEDGMENTS}

We are thankful to STAPS, University of Nantes, France for assistance in preparing and conducting the experiments.

\section{REFERENCES}

[1] D. B. Chaffin, G. B. J. Andersson, and B. J. Martin. Occupational Biomechanics. Wiley - Interscience, third edition, 1999.

[2] J. Ding, A. Wexler, and S. Binder-Macleod. Mathematical models for fatigue minimization during functional electrical simulation. journal of electromyograpgy and kinesiology, 13:575-588, 2003.

[3] J. Freund and E.-P. Takala. A dynamic model of the forearm including fatigue. Journal of Biomechanics, 34:597-605, 2001.

[4] A. Hill. The heat of shortening and dynamic constant of muscle. Proc. R. Soc. Lond. B, 126:135-195, 1938.
[5] A. Huppe, K. Muller, and H. Raspe. Is the occurrence of back pain in germany decreasing? two regional postal surveys a decade apart. European Journal of Public Health, 17:318-322, 2006.

[6] R. Lawrence, C. Helmick, F. Arnett, R. Deyo, D. Felson, E. Giannini, S. Heyse, R. Hirsch, M. Hochberg, G. Hunder, M. Liang, S. Pillemer, V. Steen, and F. Wolfe. Estimates of the prevalence of arthritis and selected musculoskeletal disorders in the united states. Arthritis \& Rheumatism, 41(5):778-799, 1998.

[7] J. Z. Liu, R. W. Brown, and G. H. Yue. A dynamical model of muscle activation, fatigue and recovery. Biophysical Journal, 82:2344-2359, 2002.

[8] L. Ma. Contributions pour l'analyse ergonomique de mannequins virtuels. $\mathrm{PhD}$ thesis, IRCCyN, Ecole Centrale de Nantes, France, 2009.

[9] L. Ma, D. Chablat, F. Bennis, and W. Zhang. A new muscle fatigue and recovery model and its ergonomics application in human simulation. Virtual and Physical Prototyping, 5:123-137, 2008.

[10] R. Ma. Modelisation de la fatigue musculaire dynamique et son application pour l'analyse ergonomique. $\mathrm{PhD}$ thesis, IRCCyN, Ecole Centrale de Nantes, 2012.

[11] R. Ma, D. Chablat, and F. Bennis. Human muscle fatigue model in dynamic motions. Latest Advances in Robot Kinematics, 0:349-356, 2012.

[12] R. Ma, D. Chablat, F. Bennis, and L. Ma. A framework of motion capture system based human behaviours simulation for ergonomic analysis. In HCI International 2011, 9-14 July, Hilton Orlando Bonnet Creek, Orlando, Florida, USA, 2011.

[13] J. M. M. Miguel T. Silva, Andre F. Pereira. An efficient muscle fatigue model for forward and inverse dynamic analysis of human movements. Procedia IUTAM, 2:262-274, 2011.

[14] O. Missenard, D. Mottet, and S. Perrey. Muscular fatigue increases signal-dependent noise during isometric force production. Neuroscience Letters, 437:154âĂŞ157, 2008.

[15] N. M. Nur, S. Z. M. Dawal, and M. Dahari. The prevelence of work related musculosceletal disorders among workers performing industrial repetitive tasks in the automotive manufacturing companies. In Proceedings of the 2014 International conference on inductrial engineering and operations management, Bali, Indonesia, 2014.

[16] W. H. Organization. Preventing musculoskeletal disorders in the workplace. Protecting workers 'Health series; no. 5', Geneva 27, Switzeland, 2003. ISBN 924159053 X.

[17] L. Punnett and D. H. Wegman. Work related musculoskeletal disorders: the epidemiologic evidence and the debate. Journal of Electromyography and Kinesiology, 14:13-23, 2004.

[18] E. V. G. Vladimir V. Syuzev, Alexander M. Gouskov. Human skeletal muscle - mechanical and mathematical models. ICABB-2010, Venice, Italy, 0:-, 2010.

[19] Vollestad and N. K. Measurement of human muscle fatigue. Journal of Neuroscience Methods, 74:219-227, 1997.

[20] T. Xia and L. A. F. Lawa. A theoretical approach for modeling peripheral muscle fatigue and recovery. Journal of Biomechanics 41 (2008) 3046âAŞ3052, 41:3046-3052, 2008. 